\title{
Evaluation of new motorized articulating laparoscopic instruments by laparoscopic novices using a standardized laparoscopic skills curriculum
}

\author{
Daniel Uysal ${ }^{1}$. Claudia Gasch ${ }^{2} \cdot$ Rouven Behnisch $^{3} \cdot$ Felix Nickel $^{4} \cdot$ Beat Peter Müller-Stich ${ }^{4}$ Markus Hohenfellner ${ }^{2}$. \\ Dogu Teber ${ }^{2,5}$
}

Received: 10 June 2020 / Accepted: 3 October 2020 / Published online: 20 October 2020

(C) The Author(s) 2020

\begin{abstract}
Background Motorized articulating laparoscopic instruments (ALI) offer more degrees of freedom than conventional laparoscopic instruments (CLI). However, a difficult learning curve and complex instrument handling are still a problem of ALI. We compared the performance of new prototypes of motorized ALI with CLI in a series of standardized laparoscopic tasks performed by laparoscopic novices. Further, usability of the new ALI was assessed.

Methods A randomized cross-over study with 50 laparoscopic novices who either started with CLI and then changed to ALI (CA) or vice versa (AC) was conducted. All participants performed the European training in basic laparoscopic urological skills (E-BLUS) with each instrument in given order. Time and errors were measured for each exercise. Instrument usability was assessed.

Results Overall, using CLI was significantly faster (CLI 4:27 min vs. ALI 4:50 min; $p$-value 0.005) and associated with fewer exercise failures in needle guidance (CLI 0 vs. ALI 12; $p$-value 0.0005) than ALI. Median amount of errors was similar for both instruments. Instrument sequence did not matter, as CA and AC showed comparable completion times. Regarding the learning effect, participants were significantly faster in the second attempt of exercises than in the first. In the needle guidance task, participants using CLI last demonstrated a significant speed improvement, whereas ALI were significantly slower in the second run. Regarding usability, CLI were preferred over ALI due to lighter weight and easier handling. Nevertheless, participants valued ALI's additional degrees of freedom.

Conclusion Using new motorized ALI in the E-BLUS examination by laparoscopic novices led to a worse performance compared to CLI. An explanation could be that participants felt overwhelmed by ALI and that ALI have an own distinct learning curve. As participants valued ALI's additional degrees of freedom, however, a future application of ALI could be for training purposes, ideally in combination with CLI.
\end{abstract}

Keywords Motorized articulating laparoscopic instruments · Handheld robotic device · Laparoscopy · E-BLUS · Urology · Training

\section{Abbreviations}

ALI Articulating laparoscopic instrument(s)

CC E-BLUS exercise "cutting a circle"

Daniel Uysal and Claudia Gasch both authors share first authorship.

Electronic supplementary material The online version of this article (https://doi.org/10.1007/s00464-020-08086-2) contains supplementary material, which is available to authorized users.

Claudia Gasch

claudia.gasch@med.uni-heidelberg.de

Extended author information available on the last page of the article
CLI Conventional laparoscopic instrument(s)

E-BLUS European training in basic laparoscopic urological skills

LS E-BLUS exercise "laparoscopic suturing"

NG E-BLUS exercise "needle guidance"

PT E-BLUS exercise "peg transfer

Within the last 40 years laparoscopic surgery has had a profound influence on the development of operative medicine. Its benefits for the patient, such as shorter hospital stay, decreased blood loss and improved cosmetic appearance have been well established [1]. The surgeon, on the other 
hand, is faced with certain difficulties like reduced perception of depth, inverse translation of movements (fulcrum effect), limited degrees of freedom and increased physical demand during surgery [2]. Therefore, surgery with conventional laparoscopic instruments (CLI) shows a more difficult learning process compared to open surgery $[3,4]$. Due to these obstacles, surgical robots, like the da Vinci ${ }^{\circledR}$ surgical system (Intuitive Surgical, Sunnyvale, California), have been developed. Robotic surgery offers a magnified 3D view and allows for a more natural instrument movement, using seven degrees of freedom as opposed to the four degrees of freedom offered by CLI. Another advantage of robotic systems is less physical strain during surgery $[5,6]$. Besides its loss of haptic feedback, potential disadvantages of robots are high acquisition and maintenance costs, allowing only for a few specialized centers to implement robotic systems into their clinical practice $[5,6]$.

To address the discrepancy of technical limitations of CLI and high costs of robotic systems, various articulating laparoscopic instruments (ALI) - mechanical or motorized-have been developed [7, 8]. The hope is that they may offer enhanced degrees of freedom while being more costeffective, thus making them affordable to a larger number of institutions. Until today only few studies tested motorized ALI. Most of them either used robotic-driven needle holders or the Kymerax ${ }^{\mathrm{TM}}$ system (Terumo, Tokyo, Japan) and were conducted in box trainers [7, 9-11]. In 2017, Sieber et al. investigated the performance of Kymerax ${ }^{\mathrm{TM}}$ instruments and found that despite increased precision participants performed tasks in a box trainer significantly more slowly and needed more training time compared to CLI [10]. Also, Tuncel's study suggests that laparoscopic suturing with firstgeneration articulating needle holder might be more difficult to learn for laparoscopic novices [12].

Since a difficult learning curve and the complex instrument handling still seem to be a problem of ALI, we wanted to test new prototypes of motorized ALI of Karl Storz (KARL STORZ SE \& Co., KG, Tuttlingen, Germany). As additional features they allow a motorized flection and $360^{\circ}$ rotation of the tip, providing more degrees of freedom than CLI (Fig. 1). For motorization they must be connected to a designated motor unit, which allows selection of different speed modes and the instrument used. In contrast to other ALI the above-mentioned features can now be performed with full seven degrees of freedom. For more intuitive usage the new ALI pistol handle combines the trigger and buttons for tip rotation and deflection on the front of the handle, instead of opposite sides of the instrument like the Kyme$\operatorname{rax}^{\mathrm{TM}}$ instruments $[7,10]$.

The aim of this study was to compare the performance of the new motorized ALI with CLI in a series of standardized laparoscopic tasks. The authors wanted to know whether the improvements in ergonomics and intuitiveness of the new
ALI translated to equal or better results than CLI. Another aim was to assess the usability of the new ALI.

\section{Material and methods}

\section{Study design}

50 medical students and residents with no prior experience in laparoscopic surgery were chosen to participate in the trial (Table 1). All participants were informed about the type and extent of this study as well as the usage of gathered data and written informed consent was obtained. The study was approved by the local Ethics Committee at Heidelberg University (Code S-334/2011). The participants were randomly assigned to one of two groups in a ratio of 1:1. Group CA started the first run of the examination with CLI and then crossed over to the motorized ALI in the second run. Group $\mathrm{AC}$ used the instruments in reverse order.

Performance with the different instruments was compared with a validated laparoscopic skills curriculum used by the European Association of Urology to certify Urology laparoscopists, the European training in basic laparoscopic urological skills (E-BLUS). The E-BLUS examination was developed to specifically evaluate the competence of basic laparoscopic skills in Urology residents and consists of four exercises: peg transfer (PT), cutting a circle (CC), needle guidance (NG), and laparoscopic suturing (LS) (Fig. 2) [13].

Our assessment started with three warm-up tasks to familiarize participants with the different instruments (Fig. 3). Afterwards, the first E-BLUS examination was started with participants watching an official exercise video tutorial prior to each exercise (https://uroweb.org/education/online-educa tion/surgical-education/laparoscopy/). A detailed explanation of the exercises according to Brinkman et al. can be found in the supplement [13]. After a cool-down period of 10 min when finishing the first run the process was repeated using the other instruments. A questionnaire regarding instrument usability was administered after each run (ALI or CLI). To account for the laparoscopic inexperience of participants, time tolerance of the exercises was adjusted. Time to completion, the number of mistakes and exercise failure were recorded for each exercise.

\section{Instrument setup}

To adequately simulate the conditions of operating in a pneumoperitoneum, all tasks took place in a box trainer model in 2D vision. Exercise boards and instruments were placed in the same position to allow for consistent distances (Fig. 4). Since only one motorized ALI could be attached to the motor unit at a time, participants were asked to use their 


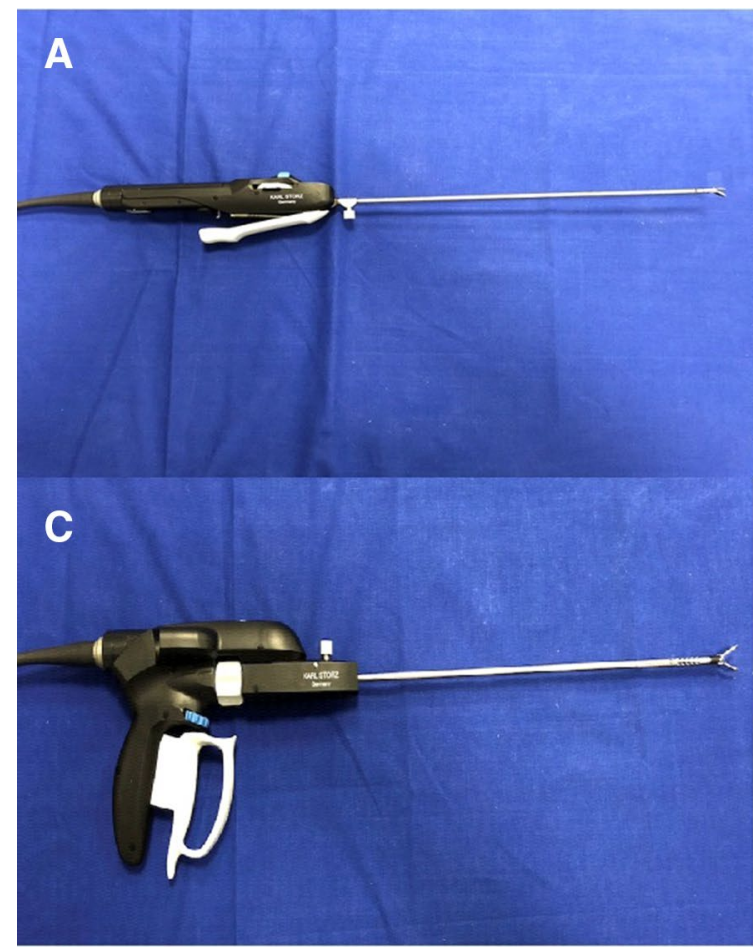

Fig. 1 A Functional sample of motorized articulating needle holder: the blue and grey wheels allow rotation and flection of the instrument tip. B Articulating needle holder's tip flexed and rotated. C

dominant hand for the motorized ALI while they were given a CLI for their non-dominant hand.

Laparoscopic vision was provided using an IMAGE1 S CONNECT and IMAGE1 S D3-Link module with a TIPCAM1 S 3D LAP $10 \mathrm{~mm}$ rigid video endoscope with a $30^{\circ}$ optic in 2D mode on a 32" TM 330 monitor (all devices by Karl Storz (KARL STORZ SE \& Co., KG, Tuttlingen, Germany). Light was provided by a cold light source POWER LED 300 (KARL STORZ SE \& Co., KG, Tuttlingen, Germany). Conventional laparoscopic instruments used were two grasping forceps (dissecting and grasping forceps), scissors and a lockable needle holder (all instruments by KARL STORZ SE \& Co., KG, Tuttlingen, Germany). Functional samples of motorized articulating instruments were provided by Karl Storz (KARL STORZ SE \& Co., KG, Tuttlingen, Germany). They consisted of a needle holder, bipolar forceps, and bipolar scissors and were connected to a designated motor unit. All devices and instruments were CE certified.

\section{Questionnaire}

Before starting the first exercises, participants were asked to provide general information. After completing the first E-BLUS examination, participants were handed a questionnaire asking about ergonomics of the instruments, exercise

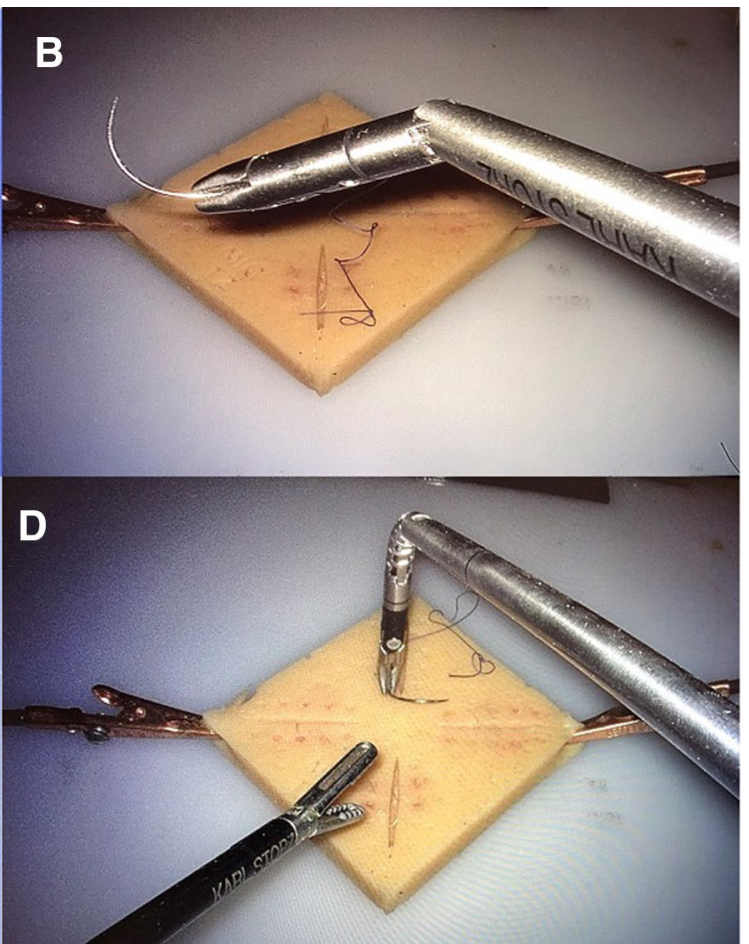

Functional sample of motorized grasping forceps: the blue and grey wheels allow rotation and flection of the instrument tip. D Articulating grasping forceps' tip flexed and rotated (Color figure online)

difficulties and any problems that occurred. A similar questionnaire with additional questions and participants` thoughts concerning features of the functional samples of motorized articulating instruments was administered after using ALI. All questionnaires were specifically designed for this study and provided in a digital format (SurveyGizmo, Widgix, LLC dba SurveyGizmo, 4888 Pearl East Cir., Suite $100 \mathrm{~W}$, Boulder, CO 80,301) on an iPad Pro® (Apple Inc, Cupertino, California, USA) as no standardized template for this purpose existed. They were added as a supplement.

\section{Statistical analysis}

Chi-Square test was used to evaluate group demographics. To compare exercise failure of CLI and ALI the McNemar test was applied. To assess time to completion for the E-BLUS exercises a mixed model was calculated with sequence, period, and instrument as fixed effects as well as a nested effect of participants within the randomized sequence to account for multiple measurements. Pairwise comparisons for each fixed effect were done in terms of least squares means. Paired $t$-tests were used to compare the time differences between the first and second run between the two sequences. All statistical analyses were performed using SAS ${ }^{\circledR}$ software v9.4 (SAS Institute Inc., Cary, NC, USA). 
Table 1 Group characteristics

\begin{tabular}{llll}
\hline & CA $n=25$ & AC $n=25$ & $p$-value \\
\hline Gender & & & 0.54 \\
Male & $16(64 \%)$ & $18(72 \%)$ & \\
Female & $9(36 \%)$ & $7(28 \%)$ & \\
Handedness & & & 1.00 \\
Right & $22(78 \%)$ & $22(78 \%)$ & \\
Left & $3(12 \%)$ & $3(12 \%)$ & \\
Age & & & 0.40 \\
$<20$ & $7(28 \%)$ & $3(12 \%)$ & \\
$20-25$ & $16(64 \%)$ & $18(72 \%)$ & \\
$26-30$ & $2(8 \%)$ & $3(12 \%)$ & \\
$>30$ & & $1(4 \%)$ & \\
Medical education & & & \\
Preclinical & $14(56 \%)$ & $7(28 \%)$ & \\
Clinical & $9(36 \%)$ & $13(52 \%)$ & \\
Internship & $2(8 \%)$ & $3(12 \%)$ & \\
Resident & & $2(8 \%)$ & \\
Hobbies & & & \\
Musical instrument & $9(36 \%)$ & $13(52 \%)$ & \\
Video gaming & $9(36 \%)$ & $6(24 \%)$ & \\
Knitting/pottery & $1(4 \%)$ & $2(8 \%)$ & \\
Suturing skills course & $1(4 \%)$ & $2(8 \%)$ & \\
\hline
\end{tabular}

Differences between groups were assessed using the Chi-Square test. A $p$-value $<0.05$ was regarded as statistically significant

Group CA Conventional laparoscopic instruments in the first, articulating laparoscopic instruments in the second run, Group AC Articulating laparoscopic instruments in the first, conventional laparoscopic instruments in the second run, $n$ number of trainees

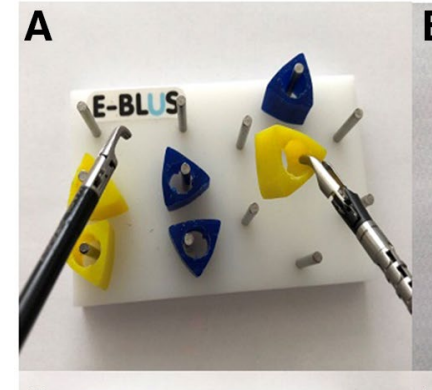

B
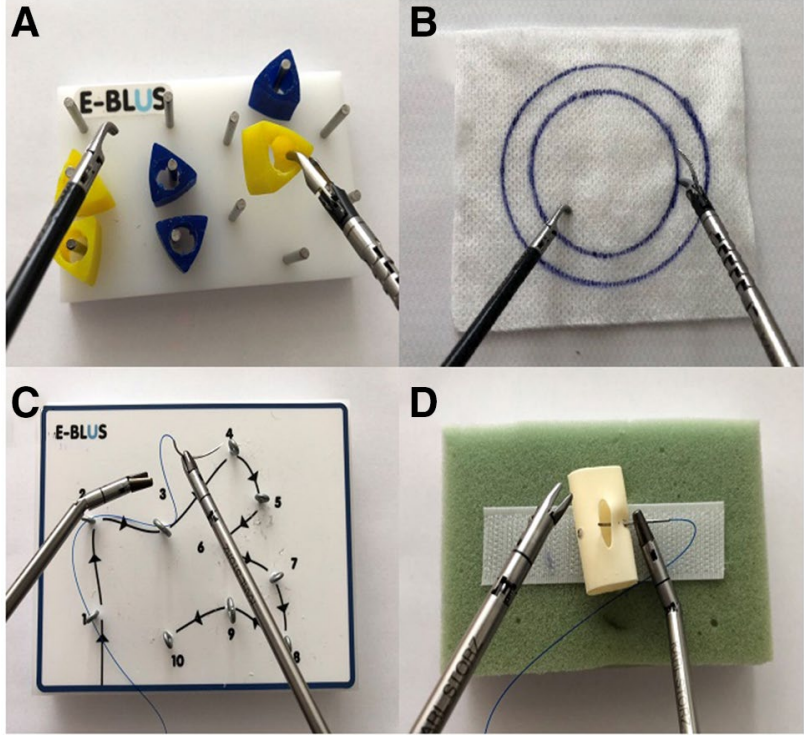

D

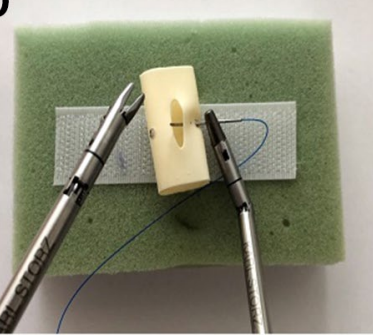

Fig. 2 The performed E-BLUS examination tasks. A Peg transfer. B Cutting a circle. C Needle guidance. D Laparoscopic suturing

\section{Results}

Participants in both groups were predominantly male, righthanded and between 20 and 25 years old. Most participants were either in their preclinical or clinical years. Detailed group characteristics can be found in Table 1. Statistical analysis revealed no significant differences between both groups.

\section{Instrument comparison (CLI vs. ALI)}

Overall, using CLI was significantly faster and associated with fewer exercise failures than the new motorized ALI, defined as not completing an exercise within the given time (Tables 2 and 3). Participants failing an exercise were excluded from further statistical analysis of this exercise. When looking at the individual exercises, however, use of ALI showed no difference for the PT and CC task. Use of CLI improved speed in the NG and LS task, which reached statistical significance in NG (Table 2). Interestingly, none of the participants failed the needle guidance task with CLI, whereas 12 attempts with ALI were not successful (Table 3).

Both ALI and CLI showed the same median amount of errors in the exercises $(1[1 ; 6]$ vs. $1[1 ; 9])$. Also, when comparing instruments for each exercise individually median errors were comparable for PT (ALI: $1[1 ; 5]$ vs. CLI: 2[1;5]), for CC (CLI: $1[1 ; 9])$ vs. ALI: $2[1 ; 6])$ and for LS $(1[1 ; 3]$ vs. $1[1 ; 3])$. No errors were made with either instrument in the NG task.

\section{Group comparison (CA vs. AC)}

When comparing the mean time needed to complete all exercises of CA and AC, it did not matter which instrument was used first, as both groups showed no difference (AC: 4:49 vs. CA: 5:02 min; $p=0.46$ ). Further, no statistical significance could be observed when regarding the time of exercises individually (PT: AC 3:09 vs. CA 3:15 min, $p=0.67$; CC: AC $2: 46$ vs. CA 2:39 min, $p=0.44$; LS: AC 6:22 vs. CA 6:16 min, $p=0.8$; NG: AC 6:12 vs. CA 6:29 min, $p=0.56$ ). Overall, the median amount of errors was similar between both groups (AC 1[1;5] vs. CA 1[1;9]).

As expected, a learning effect could be observed, since participants needed significantly less time to complete the exercises on the second attempt (P1: 4:55 vs. P2: 4:21 min; $p<0.0001)$. The same effect could be noticed for each of the individual exercises (PT: $3: 25$ vs. $2: 59 \mathrm{~min} ; p=0.004$; CC: $2: 56$ vs. $2: 29 \mathrm{~min} ; p<0.0001$; LS: $6: 44$ vs. 5:55 min; $p=0.03)$. Only the needle guidance task showed no statistical significance (6:37 vs. 6:05 $\mathrm{min} ; p=0.1)$. Interestingly, the median amount of errors was identical within both 


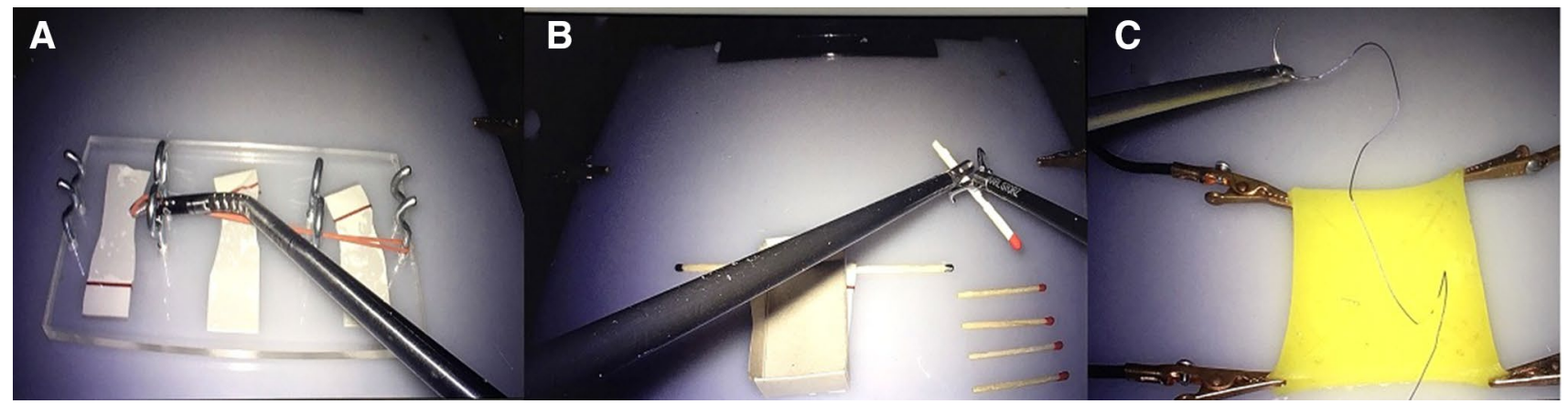

Fig. 3 Warm-up exercises. A Rubber band stretch. Articulating grasping forceps' flection allows direct advancement through the ring, which would not be possible with CLI. B Match transfer. Participants used ALI's flection to pick up matches like a crane (not depicted here). C Laparoscopic suturing. Stitches had already been placed onto the rubber pad

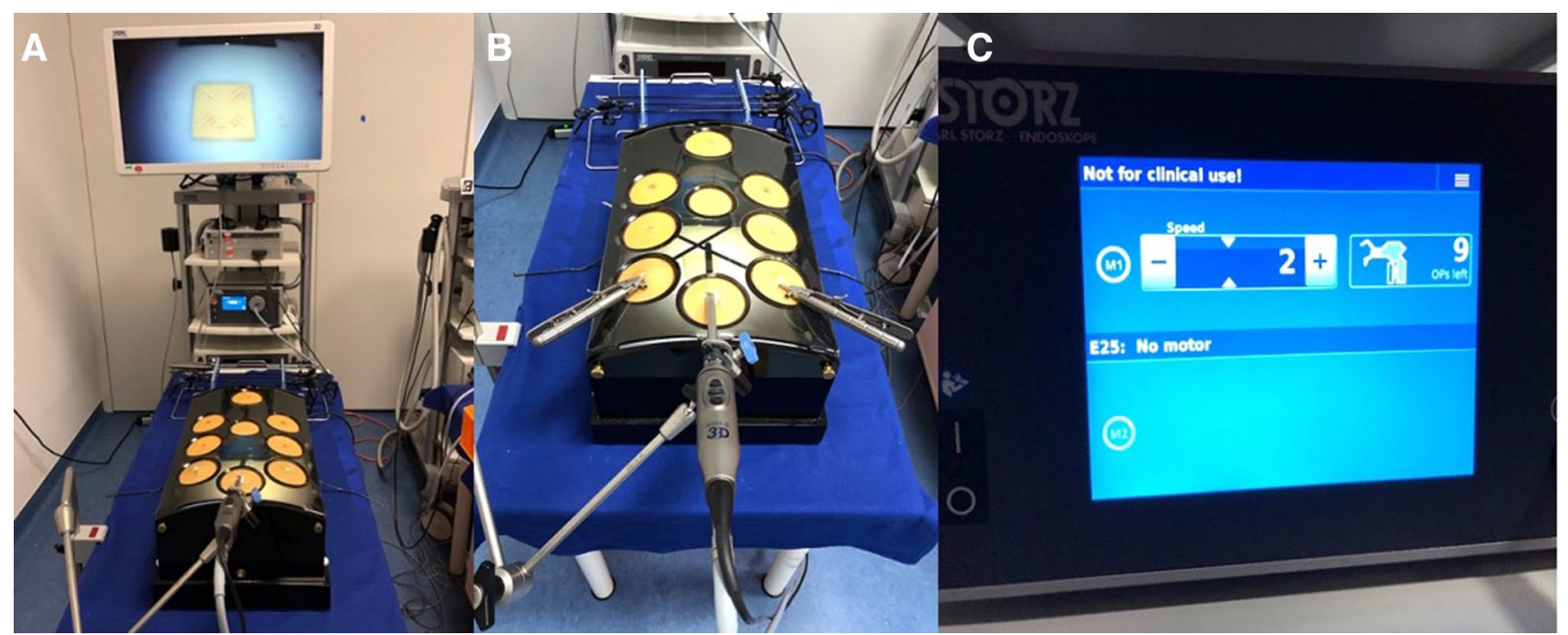

Fig. 4 Instrument setup. A Laparoscopic workstation. B Box trainer mounted with camera and two needle holders in the trocar positions used for every exercise. C Motor unit of the functional samples of articulating instruments allowing to select different instruments (right side of screen) and speed (left side of screen)
Table 2 Comparison of time needed to complete the E-BLUS exercises in respect to instrument used

\begin{tabular}{llllc}
\hline & CLI $[\mathrm{min}]$ & ALI $[\mathrm{min}]$ & ALI-CLI $(\mathrm{CI})[\mathrm{s}]$ & $p$-value \\
\hline Overall & $4: 27$ & $4: 50$ & - & 0.005 \\
Peg transfer & $3: 13$ & $3: 12$ & $-0.3(-17.5 ; 16.9)$ & 0.97 \\
Cutting a circle & $2: 44$ & $2: 40$ & $-4.0(-15.0 ; 7.0)$ & 0.47 \\
Needle guidance & $5: 36$ & $7: 05$ & $88.9(50.2 ; 127.6)$ & $<0.0001$ \\
Laparoscopic suturing & $6: 15$ & $6: 24$ & $9.1(-35.4 ; 53.7)$ & 0.68 \\
\hline
\end{tabular}

Displayed are the least square means values as well as the difference in least square means together with 95\% confidence intervals generated with the Mixed Procedure. A p-value of $<0.05$ was regarded as statistically significant

$C L I$ Conventional laparoscopic instruments, $A L I$ articulating laparoscopic instruments periods. (PT P1: $1[1 ; 5]$ vs. P2: 1[1;4]; CC P1: 2[1;9] vs. P2: 2[1;6]; LS: P1: 1[1;3] vs. P2: 1[1;3]).

When comparing both groups regarding speed improvement from the first to the second attempt, improvement in the PT task was similar (CA: 27,5 vs AC: $26,2 \mathrm{~s} ; p=0.94$ ). Although, it was not statistically significant, group CA could reduce their $\mathrm{CC}$ task time by an additional $6 \mathrm{~s}$ in the second run with ALI compared to AC (CA: $30,7 \mathrm{~s}$ vs. AC: $24,3 \mathrm{~s}$; 
Table 3 Failed attempts shown for each instrument and exercise

\begin{tabular}{llll}
\hline & $\begin{array}{l}\text { Failure CLI } \\
n=50\end{array}$ & $\begin{array}{l}\text { Failure ALI } \\
n=50\end{array}$ & $p$-value \\
\hline Peg transfer & 2 & 2 & 1.00 \\
Cutting a circle & 1 & 1 & 1.00 \\
Needle guidance & 0 & 12 & 0.0005 \\
Laparoscopic suturing & 7 & 13 & 0.06 \\
\hline
\end{tabular}

A p-value of $<0.05$ was regarded as statistically significant

$C L I$ Conventional laparoscopic instruments, $A L I$ articulating laparoscopic instruments, $n$ number of trainees

$p=0.56)$. Participants were clearly faster when using CLI last (group AC) in LS and NG and showed a great speed improvement from the first to the second attempt compared to CA (LS: AC $86,6 \mathrm{~s}$ vs. CA $51,8 \mathrm{~s}, p=0.45$ and NG: AC $136,2 \mathrm{~s}$ vs. $\mathrm{CA}-70,1 \mathrm{~s}, p<0.0001$,). Interestingly, group $\mathrm{AC}$ was $136,2 \mathrm{~s}$ faster in the second run of the NG task, while no improvement for this exercise could be seen in the CA group at all. Participants using the new ALI after CLI even showed a statistically significant slower performance in the second attempt (Fig. 5).

\section{Usability}

In general, participants preferred CLI over the new motorized ALI due to its lighter weight and easier handling. Opening and closing of ALI was associated with slightly more discomfort with a minority of trainees even stating major discomfort (Fig. 6A). Fit of grip and hand dimensions as well as grip comfort for the needle holder and the grasping forceps were almost similar for both instruments with a minority of students again reporting no fit at all or the grip being very uncomfortable for ALI, especially for the needle holder (Fig. 6B, C). Interestingly, grip precision was excellent in both groups with ALI allowing slightly more participants very precise work for the entire time (Fig. 6D).

$61 \%$ of participants believed that additional degrees of freedom of ALI allowed easier knot tying and suturing compared to CLI, since angulating the tip from the shaft facilitated knot tying and handling of the suture. 29\% disagreed, stating that ALI was too heavy and that additional degrees of freedom made the exercise more complicated. When asked if the possibility to angulate the tip of the scissors facilitated the cutting a circle task, $88 \%$ agreed stating that the biggest advantage of ALI over CLI could be seen within this exercise. $94 \%$ of trainees thought using ALI could improve working in small or narrow spaces, although some believed further experience with ALI would be needed. Further, $90 \%$ believed ALI could facilitate working around objects such as vessels. $73 \%$ agreed that ALI could offer additional benefit for knot tying and suturing in sloped planes such as the psoas muscle while $14 \%$ opposed.

Often encountered problems using ALI were heavy instruments making precise movements no longer possible, unergonomic and difficult to use ALI needle holder and the thread getting stuck in the hinge during knot tying. Overall, participants did not approve of the new ALI.

\section{Discussion}

Over the last decades mechanical and motorized ALI have been developed to help the surgeon overcome the known obstacles of laparoscopy and, thus, making more difficult procedures possible in a minimally invasive manner. But since their introduction few studies have compared motorized ALI to CLI and their benefit is still unclear [7, 9-11, 14-16]. Some studies only compared tasks performed with motorized needle holders, like Robot DEX ${ }^{\mathrm{TM}}$ (Déxtérité Surgical, Annecy, France) or Jaimy ${ }^{\circledR}$ (Endocontrol Company, Grenoble, France) $[11,15,16]$, whereas others also used several instruments of the Kymerax ${ }^{\mathrm{TM}}$ system (Terumo, Tokyo, Japan) comparable to our study $[9,10,14,17]$. In this trial, we evaluated the performance of new motorized ALI of Karl Storz (KARL STORZ SE \& Co., KG, Tuttlingen, Germany), consisting of bipolar scissors, bipolar forceps, and needle holder, in comparison to CLI. All of the above mentioned Kymerax $^{\mathrm{TM}}$ studies investigated ALI's performance in different levels of proficiency (novice, intermediate, expert) and participants per group reached up to a maximum of 25 [9, $10,14,17]$. In contrast, we wanted to assess the new motorized ALI in a larger cohort of laparoscopic novices to create a homogenous and objective study population and, thus, no potential bias towards CLI as shown in other studies $[9,15]$.

Our results show that using CLI in the E-BLUS examination was significantly faster and associated with fewer exercise failures than the new motorized ALI. One explanation could be that participants felt overwhelmed by ALI's additional degrees of freedom and instrument handling combined with the well-known intricacies of laparoscopic surgery. This is further supported by the fact that ALI showed a worse performance in more challenging tasks like NG or LS, as they require a greater depth perception and more complex instrument maneuvers.

These findings are in concordance with above mentioned research of Sieber et al. who found that participants were slower and needed more training time when using Kyme$\operatorname{rax}^{\mathrm{TM}}$ compared to CLI. Their tasks were also performed in a box trainer and comparable to the ones in our study. In contrast to our results, an improved accuracy with ALI use for all exercises was recorded [10]. Our results showed a similar error rate for ALI and CLI in all tasks. It must be 


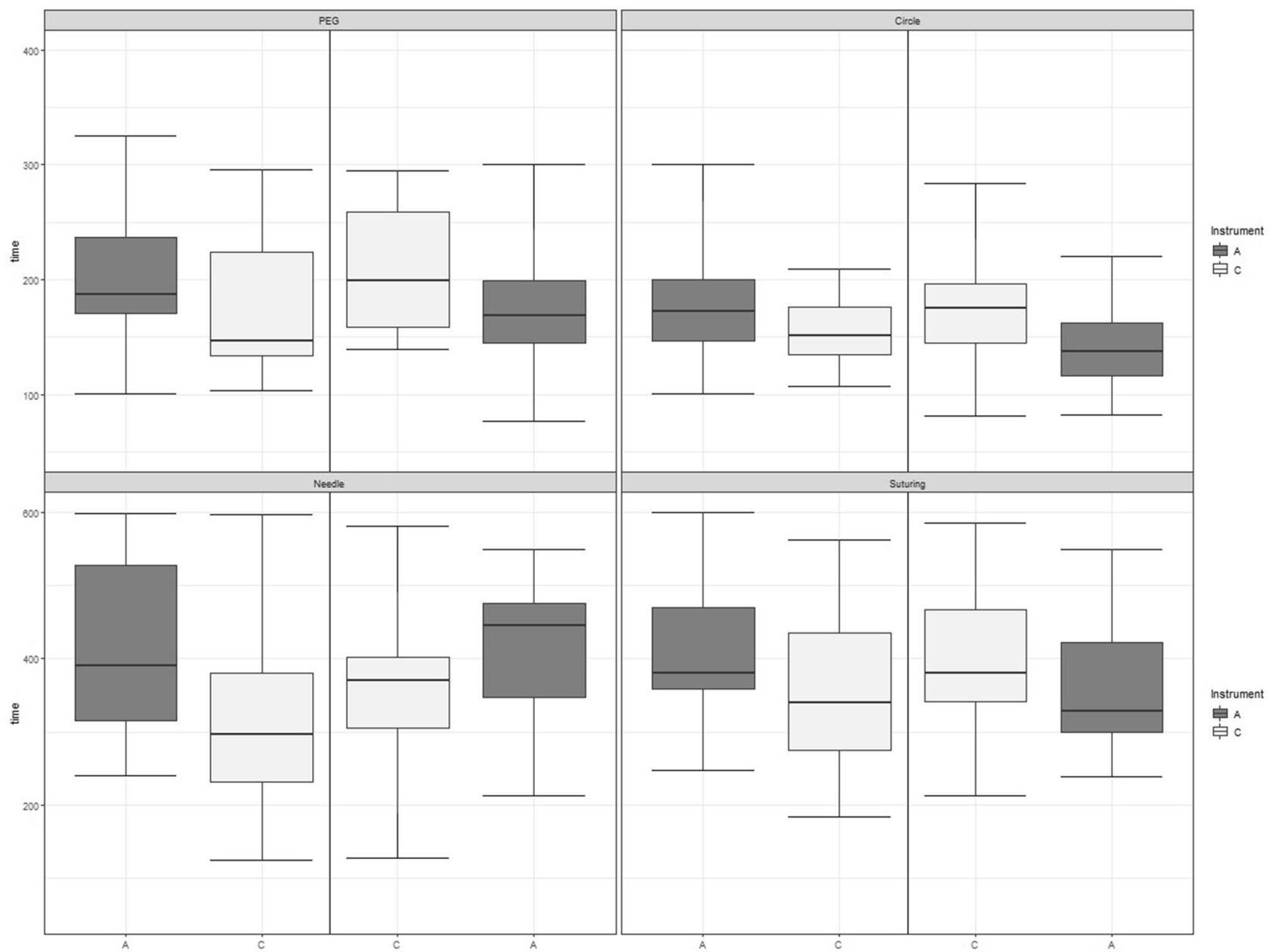

Fig. 5 Time change from first to second attempt of E-BLUS exercises compared between groups AC and CA. Boxplots show the median time and range (in sec) of ALI and CLI for each exercise stratified by groups. Note the statistically significant slower performance for

noted however, that Sieber et al. used a "fraying factor" for assessing instrument accuracy in cutting tasks, whereas fraying in the cutting task in our trial was not assessed as strictly leading to limited transferability of results [10].

Regarding the skills assessment in our study, it must be mentioned that only time, errors, and exercise failure were observed as foreseen in the original E-BLUS examination. However, this may have confounded our study as we were probably not able to observe the full potential of the new ALI. Especially, when evaluating more complex instruments such as motorized ALI, it would make sense to integrate tissue damage parameters such as instrument movement and interaction force into the skill assessment [18]. In a recent study about force-based laparoscopic skills training, the authors state that it is advisable to first train surgeons on efficient tissue manipulation and instrument handling before allowing them to focus on efficiency of time [19]. the needle guidance task at the second attempt in group CA. (A) ALIArticulating laparoscopic instruments. (C) CLI Conventional laparoscopic instruments. PEG Peg transfer, Circle Cutting a circle, Needle Needle guidance, Suturing Laparoscopic suturing

In this way, when training with ALI, safe tissue handling, and instrument accuracy could be evaluated more precisely.

Although there are a lot of laparoscopic tasks, we chose to use the E-BLUS examination as a validated basic laparoscopic skills curriculum in our study for reproducibility and transferability of tasks. Additionally, the tasks were selected based on the possibility to complete them with both types of instruments (ALI and CLI). Further, our study cohort only consisted of laparoscopic novices. Since E-BLUS tasks are designed to teach basic laparoscopic skills to young urology residents worldwide, they could therefore easily be transferred to our participants [13]. Furthermore, the E-BLUS examination had already been used with other ALI and showed promising results in beginners [14]. However, using only four basic laparoscopic tasks could be a limitation of our study since the E-BLUS examination was especially designed for the ergonomics of CLI and not for 

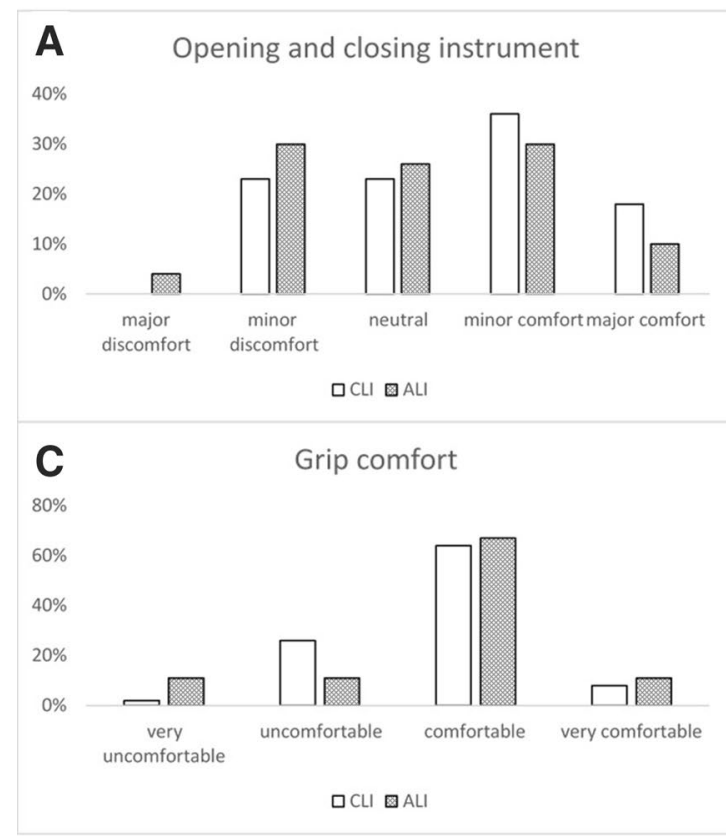

Fig. 6 Questionnaire results of usability of instruments. Displayed are the percentages of participants' opinions regarding opening and closing, grip and hand dimensions, grip comfort and grip precision of

ALI [13]. Most advantages of motorized ALI are seen in more challenging tasks like suturing in difficult angles as seen in performing urethrovesical anastomosis or suturing on organic tissue, i.e. during bowel surgery or partial nephrectomies. Therefore, it would be interesting to explore whether the functionality of the new motorized ALI could be more accurately assessed by more demanding exercises like a "3D pick-and-place task" or ex vivo animal models in further studies $[8,11,17,20]$. We chose laparoscopic beginners for our study cohort to ensure that we would obtain a large and consistent study population. However, some studies suggest that most advantages of ALI are only seen in experienced laparoscopist [10, 14, 21]. Therefore, it would also be of great interest to see if more experienced surgeons could expand their surgical skills by using the new ALI of our study.

Instrument usability could have played another role in differences of exercise performance in our trial. Participants generally preferred CLI over motorized ALI due to their lighter weight and easier handling. However, the ALI scissors showed promise for circular cutting tasks, as a speed improvement could be noticed in all groups and $88 \%$ of participants agreed that the biggest advantage of ALI over CLI could be seen within this exercise. A promising clinical application of ALI could therefore lie within circular cutting tasks, like partial nephrectomies, as already shown by laparoendoscopic single-site surgery using Kymerax ${ }^{\mathrm{TM}}$ instruments in a porcine model [17].
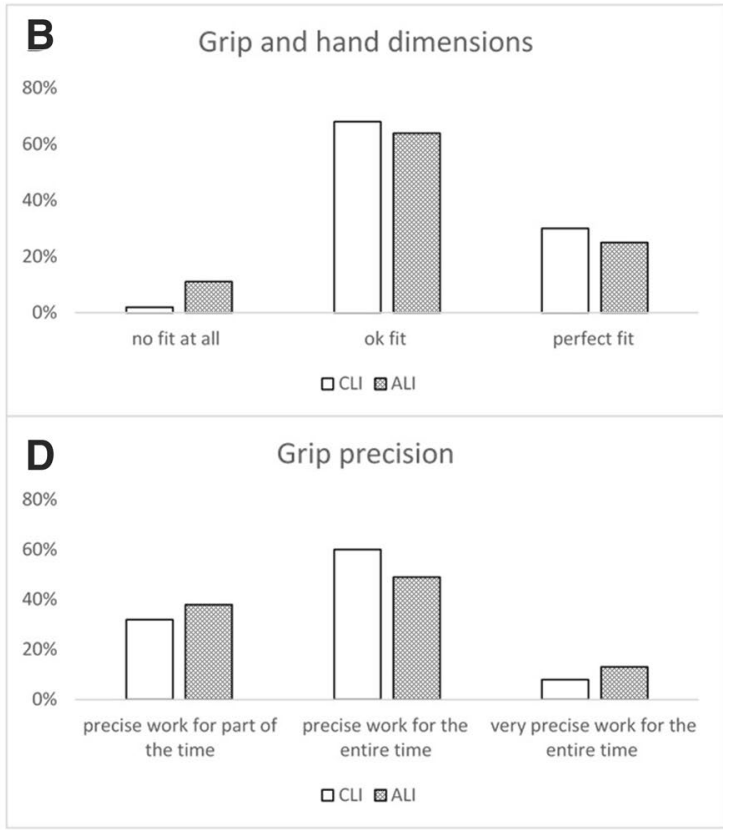

instruments. CLI Conventional laparoscopic instruments, ALI Articulating laparoscopic instruments

In contrast, many participants criticized the ALI needle holder for its opening/closing mechanism and uncomfortable grip. Since the ALI needle holder was used in the NG and LS task, the worse performance of ALI in those exercises could be explained. Although, participants performed LS clearly slower with ALI in our study, surprisingly $61 \%$ still believed that the additional degrees of freedom allowed easier knot tying and suturing compared to CLI. Therefore, a potential future application of ALI would be for training purposes of laparoscopic novices if instrument features could be improved further. This is supported by Zapardiel et al. who tested Kymerax ${ }^{\mathrm{TM}}$ instruments in physicians with different laparoscopic experience. He found that using ALI could help beginners in training because they needed less time for needle loading and placing a stitch with ALI compared to CLI. However, this advantage could not be seen in physicians with laparoscopic experience as they are already used to CLI [9]. In concordance, another study found that novices outscored experts in terms of net improvement in performance with articulating instruments. To speed the learning process, the authors suggest the use of articulating instruments at an early stage of surgical training [21]. Thus, follows that ALI seem to have their own learning curve and require skills distinct form conventional laparoscopy or open surgery. However, one should keep in mind that ALI are not designed to be a replacement of CLI, but should rather be seen as an add-on to refine laparoscopic capabilities [7]. Therefore, laparoscopic training with CLI and ALI should 
ideally be combined in a new multi-modality training curriculum to ensure an optimal training benefit with both types of instruments. One approach could be comparable to the multi-modality training for laparoscopic cholecystectomy which has already been explored by Kowaleski and colleagues [22].

\section{Conclusion}

In conclusion, using new motorized ALI in a basic laparoscopic skills curriculum by beginners was associated with a slower performance and more exercise failures compared to CLI. One explanation could be that laparoscopic novices felt overwhelmed by the additional features of motorized ALI and ALI have a distinct learning curve that needs to be addressed. However, participants believed that additional degrees of freedom allowed easier knot-tying, suturing, and cutting compared to CLI. Therefore, ALI should be used for laparoscopic training purposes, ideally combined with conventional laparoscopic training in a new curriculum. Enhancing ergonomics and reducing weight could help to further improve ALI's user-friendliness.

Acknowledgements Many thanks to all participants and to Karl Storz SE \& Co. KG for providing the functional samples of motorized articulating laparoscopic instruments.

Funding Open Access funding enabled and organized by Projekt DEAL.

\section{Compliance with ethical standards}

Disclosures For the duration of this study the samples of motorized articulating laparoscopic instruments were provided by Karl Storz SE \& Co. KG, no financial funding was received. Felix Nickel reports receiving travel support for conference participation as well as equipment provided for laparoscopic surgery courses by Karl Storz SE \& Co. KG, Johnson \& Johnson, Intuitive Surgical, Cambridge Medical Robotics, and Medtronic and he reports receiving consultancy fees from Karl Storz SE \& Co. KG. Otherwise, the authors Daniel Uysal, Claudia Gasch, Rouven Behnisch, Beat Peter Müller-Stich, Markus Hohenfellner and Dogu Teber have no conflicts of interest or financial ties to disclose.

Open Access This article is licensed under a Creative Commons Attribution 4.0 International License, which permits use, sharing, adaptation, distribution and reproduction in any medium or format, as long as you give appropriate credit to the original author(s) and the source, provide a link to the Creative Commons licence, and indicate if changes were made. The images or other third party material in this article are included in the article's Creative Commons licence, unless indicated otherwise in a credit line to the material. If material is not included in the article's Creative Commons licence and your intended use is not permitted by statutory regulation or exceeds the permitted use, you will need to obtain permission directly from the copyright holder. To view a copy of this licence, visit http://creativecommons.org/licenses/by/4.0/.

\section{References}

1. Demir A, Günseren KÖ, Kordan Y, Yavaşçaoğlu İ, Vuruşkan BA, Vuruşkan H (2016) Open vsLaparoscopic simple prostatectomy: a comparison of initial outcomes and cost. J Endourol 30:884-889. https://doi.org/10.1089/end.2016.0261

2. Supe AN, Kulkarni GV, Supe PA (2010) Ergonomics in laparoscopic surgery. J Minim Access Surg 6:31-36

3. Figert PL, Park AE, Witzke DB, Schwartz RW (2001) Transfer of training in acquiring laparoscopic skills. J Am Coll Surg 193:533-537. https://doi.org/10.1016/S1072-7515(01)01069-9

4. Romero P, Brands O, Nickel F, Müller B, Günther P, HollandCunz S (2014) Intracorporal suturing-driving license necessary? J Pediatr Surg 49:1138-1141. https://doi.org/10.1016/J. JPEDSURG.2013.12.018

5. Atug F, Castle EP, Woods M, Davis R, Thomas R (2006) Robotics in urologic surgery: an evolving new technology. Int J Urol 13:857-863. https://doi.org/10.1111/j.1442-2042.2006.01428.x

6. Yohannes P, Rotariu P, Pinto P, Smith AD, Lee BR (2002) Comparison of robotic versus laparoscopic skills: is there a difference in the learning curve? Urology 60:39-45. https://doi. org/10.1016/s0090-4295(02)01717-x

7. Sánchez-Margallo FM, Sánchez-Margallo JA, Szold A (2018) Handheld devices for laparoscopic surgery. In: Ferhatoglu MF (ed) New horizons in laparoscopic surgery. IntechOpen, London

8. Hardon SF, Schilder F, Bonjer J, Dankelman J, Horeman T (2019) A new modular mechanism that allows full detachability and cleaning of steerable laparoscopic instruments. Surg Endosc 33:3484-3493. https://doi.org/10.1007/s00464-019-06849-0

9. Zapardiel I, Hernandez A, De Santiago J (2015) The efficacy of robotic driven handheld instruments for the acquisition of basic laparoscopic suturing skills. Eur J Obstet Gynecol Reprod Biol 186:106-109. https://doi.org/10.1016/J.EJOGRB.2015.01.013

10. Sieber MA, Fellmann-Fischer B, Mueller M (2017) Performance of Kymerax@precision-drive articulating surgical system compared to conventional laparoscopic instruments in a pelvitrainer model. Surg Endosc 31:4298-4308. https://doi.org/10.1007/ s00464-017-5438-8

11. Sánchez-Margallo JA, Sánchez-Margallo FM (2017) Initial experience using a robotic-driven laparoscopic needle holder with ergonomic handle: assessment of surgeons' task performance and ergonomics. Int J Comput Assist Radiol Surg 12:2069-2077. https://doi.org/10.1007/s11548-017-1636-Z

12. Tuncel A, Lucas S, Bensalah K, Zeltser IS, Jenkins A, Saeedi O, Park S, Cadeddu JA (2008) A randomized comparison of conventional vs articulating laparoscopic needle-drivers for performing standardized suturing tasks by laparoscopy-naive subjects. BJU Int 101:727-730. https://doi.org/10.1111/j.1464410X.2007.07220.x

13. Brinkman WM, Tjiam IM, Schout BMA, Muijtjens AMM, Van Cleynenbreugel B, Koldewijn EL, Alfred Witjes J (2014) Results of the European basic laparoscopic urological skills examination. Eur Urol 65:490-496. https://doi.org/10.1016/j. eururo.2013.10.036

14. Aykan S, Akin Y, Pelit ES, Gulmez H, Tuken M, Colakerol A, Semercioz A, Muslumanoglu AY (2017) Impact of motorized articulating laparoscopic devices with three-dimension visualizing system: a pilot study. J Endourol 31:174-179. https://doi. org/10.1089/end.2016.0406

15. Leite M, Carvalho AF, Costa P, Pereira R, Moreira A, Rodrigues N, Laureano S, Correia-Pinto J, Vilaça JL, Leão P (2016) Assessment of laparoscopic skills performance. Surg Innov 23:52-61. https://doi.org/10.1177/1553350615585638

16. Bensignor T, Morel G, Reversat D, Fuks D, Gayet B (2016) Evaluation of the effect of a laparoscopic robotized needle 
holder on ergonomics and skills. Surg Endosc 30:446-454. https ://doi.org/10.1007/s00464-015-4217-7

17. Sánchez-Margallo FM, Sánchez-Margallo JA (2018) Assessment of postural ergonomics and surgical performance in laparoendoscopic single-site surgery using a handheld robotic device. Surg Innov 25:208-217. https://doi.org/10.1177/1553350618 759768

18. Horeman T, Rodrigues SP, Willem Jansen F, Dankelman J, van den Dobbelsteen JJ (2012) Force parameters for skills assessment in laparoscopy. IEEE Trans Haptics 5:312-322. https://doi. org/10.1109/TOH.2011.60

19. Hardon SF, Horeman T, Bonjer HJ, Meijerink WJHJ (2018) Forcebased learning curve tracking in fundamental laparoscopic skills training. Surg Endosc 32:3609-3621. https://doi.org/10.1007/ s00464-018-6090-7

20. Vigneswaran HT, Dobbs RW, Huang J, Sofer LA, Halgrimson WR, Crivellaro S (2019) Use of a novel articulating laparoscopic needle driver for partial nephrectomy: an initial experience.
Urology 132:123-129. https://doi.org/10.1016/J.UROLO GY.2019.05.049

21. Martinec DV, Gatta P, Zheng B, Denk PM, Swanström LL (2009) The trade-off between flexibility and maneuverability: task performance with articulating laparoscopic instruments. Surg Endosc 23:2697-2701. https://doi.org/10.1007/s00464-009-0462-y

22. Kowalewski KF, Garrow CR, Proctor T, Preukschas AA, Friedrich M, Müller PC, Kenngott HG, Fischer L, Müller-Stich BP, Nickel F (2018) LapTrain: multi-modality training curriculum for laparoscopic cholecystectomy - results of a randomized controlled trial. Surg Endosc 32:3830-3838. https://doi.org/10.1007/s0046 4-018-6110-7

Publisher's Note Springer Nature remains neutral with regard to jurisdictional claims in published maps and institutional affiliations.

\section{Affiliations}

\section{Daniel Uysal ${ }^{1}$. Claudia Gasch ${ }^{2} \cdot$ Rouven Behnisch $^{3} \cdot$ Felix Nickel $^{4} \cdot$ Beat Peter Müller-Stich ${ }^{4}$ Markus Hohenfellner $^{2}$. Dogu Teber ${ }^{2,5}$}

1 Medical School, University of Frankfurt, Frankfurt, Germany

2 Department of Urology, Heidelberg University Hospital, Im Neuenheimer Feld 420, 69120 Heidelberg, Germany

3 Institute of Medical Biometry and Informatics (IMBI), University of Heidelberg, Heidelberg, Germany
4 Department of General, Visceral, and Transplantation Surgery, Heidelberg University Hospital, Heidelberg, Germany

5 Department of Urology, Staedtisches Klinikum Karlsruhe, Karlsruhe, Germany 